\title{
Synthesis and Characterization of Hydroxyapatite Nanoparticles using Wet Chemical Method
}

\author{
V. Kalaiselvi*1, 2 , R. Mathammal ${ }^{3}$ and P. Anitha ${ }^{4}$ \\ ${ }^{1}$ Department of Physics, Sri Sarada college for women, Salem, India \\ 2Department of Physics, Navarasam Arts \& Science College for Women, Arachalur, Tamil Nadu \\ ${ }^{3}$ Department of Physics, Sri Sarada College for women, Salem, Tamil Nadu \\ ${ }^{4}$ Department of Physics, Vellalar College for women, Erode, Tamil Nadu
}

ABSTRACT: Calcium Phosphate is an attractive biomedical materials owing to their excellent biocompatibility and nontoxicity of their chemical components. It exists in different crystalline phases and different $\mathrm{Ca} / \mathrm{P}$ ratios, such as Hydroxyapatite, Octacalcium phosphate and Tricalcium phosphate. Among the Calcium Phosphate, Hydroxyapatite Ca 10 $\left(\mathrm{PO}_{4}\right)_{6}(\mathrm{OH})_{2}$ has been used to manufacture biomaterials of hard tissue repair and replacement. In the present work, hydroxyapatite was synthesized using wet chemical method. The synthesized samples were characterized by X-ray diffraction (XRD), scanning electron microscopy (SEM) and Fourier transform infrared spectroscopy (FTIR). X- ray diffraction (XRD) analysis show that the synthesized samples appeared in single phase Hexagonal structure. The crystallite size increases from $10 \mathrm{~nm}$ to $50 \mathrm{~nm}$ with respect to increment of calcined temperature from 400 to $700 \mathrm{C}$. FTIR spectra reveal the presence of characteristic phosphate, hydroxide and water bands of Hydroxyapatite in all the prepared samples. The SEM analysis shows the formation of nanorod morphology. Results from the above mentioned characterization predicts that increment of calcinations temperature enhanced the crystalline nature and crystalline size of the nanoparticles.

KEYWORDS: Hydroxyapatite, Calcination, wet chemical method, XRD, SEM, FT-IR.

(C) 2017 mahendrapublications.com, All rights reserved

\section{INTRODUCTION}

Hydroxyapatite is known as the mineral component of the bone. Bone is an organ that not only provides a basic mechanical support to the body by generating and transferring forces that are involved in the locomotion but also has various other functions [1]. The natural and synthetic material has been developed for Biomaterials applications. The biomaterial fabrication involves restoration of body tissue functioning, mechanical properties, design and its biocompatibility. The biomaterial should possess the following mechanical properties namely elasticity, wear resistance, yield stress, toughness and ductility etc [2] It should be formed in many shapes, low cost and easy availability. They are applied in fabrication of implant devices such as hip joint prosthesis, joint prosthesis, dental implants. Biomaterials are nontoxic, biocompatible and bioactive, integrate into living tissue. Hydroxyapatite, a calcium phosphate material in crystalline form $\mathrm{Ca}_{10}\left(\mathrm{PO}_{4}\right)_{6}(\mathrm{OH})_{2}$. Bone is a fiberreinforced composite, teeth is calcium orthophosphatebased calcified phase. Teeth has two important minerals namely enamel and dentin [3]. The crystal known forms are HAp is monoclinic(P21/b) and hexagonal (P63/m) with $\mathrm{Ca}_{10}\left(\mathrm{PO}_{4}\right)_{6}(\mathrm{OH})_{2}$.Hydroxyapatite shows excellent antibacterial properties when incorporate with silver ions due to interaction of silver ions with thiol groups[4]. Bone consist of approximately $8 \mathrm{wt} \%$ water, $22 \mathrm{wt} \%$ protein and $70 \mathrm{wt} \%$ minerals. The mineral component of the bone is a form of calcium phosphate which presents the main mineral reservoir for the body. Among all biomaterials calcium phosphate are attractive biomaterials owing to its excellent biocompatibility and its non- toxicity. It is a very good drug delivery carrier and possesses favorable biodegradability and biocompatibility properties [5]. Hydroxyapatite is soluble and less toxic than silica, Quantum dots, carbon nanotubes and magnetic particles. Clacium phosphate based system and particularly those with $\mathrm{Ca} / \mathrm{P}$ molar ratio close to the one of hydroxyapatite are negligibly soluble in blood which is by itself supersaturated with respect to hydroxyapatite [6]. Calcium phosphate exists in different forms according to $\mathrm{Ca} / \mathrm{P}$ ratio, such as Hydroxyapatite, Octacalcium phosphate, tricalcium phosphate, dicalcium phosphate dehydrate and dicalcium phosphate [7]. Among these Hydroxyapatite with hexagonal crystalline structure accounts $65 \mathrm{wt} \%$ of bone and provides most of its strength and stiffness. Hydroxyapatite crystals in bone are generally in the form of rod like crystals in the nanometre size range of less than $100 \mathrm{~nm}$. Also this Hydroxyapatite has excellent biocompatibility and Osteoconductivity [8]. This property of Osseo integration is needed to minimize discharge to surrounding tissues and to increase the implant efficiency. The preparation of Hydroxyapatite powder with controlled morphology, stoichiometry, crystalline and crystallite size in nano range has the main role in the production of biomaterials. The main limitation of Hydroxyapatite bio ceramic is that it has poor mechanical properties. Hydroxyapatite on the other hand has high bioactivity, nontoxic with many medical applications in the form of porous dense and granular forms [9]. Several different

*Corresponding Author: nk.arthi.kalai@gmail.com and mathammals_shanmugam@yahoo.com

Received: 11.09 .2017

Accepted: 15.11 .2017

Published on: 12.12.2017

Kalaiselvi et al., 
Hydroxyapatite synthesis techniques have been developed in the recent years. These techniques include CoPrecipitation, wet chemical, spray pyrolysis, hydrothermal synthesis, emulsion processing, and mechano chemical method [10].

Of all these methods wet chemical method has received more attention because of inherent advantages such as homogeneous molecular mixing, low processing temperature, ability to produce nano crystalline powders and high purity [11]. This route can be used to produce very sophisticated nanomaterial and to tailor the materials to very specific applications. The wet chemical product characterized by nano size dimension is on very important parameter to improve the contact and stability at the artificial/ natural bone interface [12].

The aim of the present study is to synthesis and to determine the crystallite size, surface morphology in the presence of functional groups in the prepared Hydroxyapatite sample with different calcinations temperature from $400^{\circ} \mathrm{C}$ to $700^{\circ} \mathrm{C}$. The samples were characterized by X-Ray diffraction (XRD), Fourier transform infrared spectroscopy (FT-IR) and scanning electron microscopy (SEM) analysis respectively and the results were reported.

\section{MATERIALS AND METHODS}

Calcium hydroxide and Orthophosphoric acid were taken as the starting calcium and phosphorous precursors. $1 \mathrm{M}$ of calcium hydroxide and $0.6 \mathrm{M}$ of Orthophosphoric acid were dispersed in double distilled water and it was stirred for half-an-hour separately. Then Orthophosphoric acid solution was added drop wise to calcium hydroxide solution. After mixing it the precipitated solution was vigorously stirred for one hour. Aqueous ammonia was added drop wise to change the $\mathrm{pH}$ until the $\mathrm{pH}$ maintain at 11. The mixed solution was kept in ice for 22 hours. The precipitated solution was dried at $200^{\circ} \mathrm{C}$ for 6 hours in a dried oven. The dried powder was collected and calcined at different temperature ranging from $400-700^{\circ} \mathrm{C}$ for 3 hours. The powders were labeled as powder calcined at $400^{\circ} \mathrm{C}$ as $\mathrm{HAP} 1,500^{\circ} \mathrm{C}$ as HAP2, $600^{\circ} \mathrm{C}$ as HAP 3 and $700^{\circ} \mathrm{Cas}$ HAP 4 respectively.

\section{CHARACTERIZATION METHODS}

$\mathrm{X}$ - ray diffraction (XRD) analysis was performed for all the samples. The data were recorded in between $20^{\circ}$ to $80^{\circ}$. The XRD was performed in Bruker D8 Advance Diffractometer. It has one dimensional detector performed in integrated mode. It is nickel filtered with $\mathrm{CuK} \alpha$ radiation [13]. (Make - Shimadzu, Model - XRD 6000, Japan). The Fourier transform infrared (FT-IR) Spectra were recorded on a Perkin Elmer Spectrometer, in the range of $400 \mathrm{~cm}^{-1}$ to $4000 \mathrm{~cm}^{-1}$ [14] was carried out to identify the functional groups present in the synthesized nanoparticle. The shape and surface morphology were identified using scanning electron microscopy (Make - JEOL, Model - JSM 6390, Made in Japan).

\section{RESULTS AND DISCUSSION}

Figure 1, shows the XRD pattern of Hydroxyapatite phase evolution for HAP1, HAP2, HAP3 AND HAP4. All spectra show single phase Hexagonal structure without secondary formations. The powders were found to be hexagonal and have matched with JCPDS card no. 09-0432. The Hydroxyapatite crystallinity seems to increase with the increasing calcinations temperature. The amount of crystallinity and crystalline size for different calcined temperature was determined using Debye Scherrer equation and it is given in the Table 1 . The crystallite size is calculated from three different $2 \theta$ peaks such as $25.78^{\circ}$, $31.95^{\circ}, 46.72^{0}$ having hkl values (002), (211), (222) respectively are well separated by having high intensities were chosen for the calculations. The average crystallite size of all samples was summarized in the table 1.The crystallite size has increased from $10 \mathrm{~nm}$ and $57 \mathrm{~nm}$ for HAP 1 to HAP 4 nanopowders.

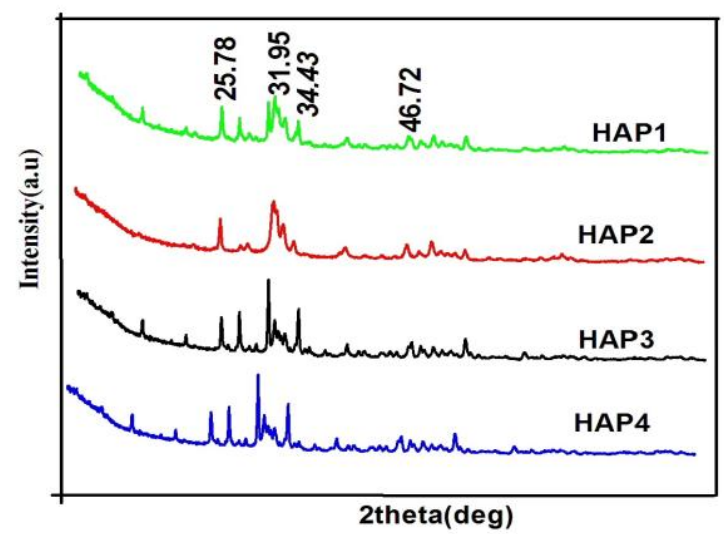

\section{Fig:1 XRD spectra of HAP1, HAP2, HAP3 and HAP4 powders}

The crystallinity degree of the calcined samples corresponding to the fraction of crystallite phase present in the examined volume was evaluated by the above formula. The mean Crystallite size of the particle was calculated from the XRD line broadening measurement from the Debye Scherrer equation

$$
\mathrm{D}=0.89 \lambda / \beta \cos \theta
$$

Where $\lambda$ is the wave length of the X-ray, $\beta$ is the FWHM and $\theta$ is the diffraction angle.

The degree of crystallinity of the calcined powders was evaluated by

$$
X_{c}=1-V_{112 / 300} / I_{300}
$$

Where $I_{300}$ is the intensity of (300) reflection of hydroxyapatite,

$V_{112 / 300}$ is the intensity of the hollow between (112) and (300) reflections.

Table 1

\begin{tabular}{|c|c|c|c|}
\hline S.No & Sample & $\begin{array}{c}\text { Crysatllite } \\
\text { size }\end{array}$ & $\begin{array}{c}\text { Degree of } \\
\text { Crystallinity }\end{array}$ \\
\hline 1 & HAP1 & $10 \mathrm{~nm}$ & 0.266 \\
\hline 2 & HAP2 & $17 \mathrm{~nm}$ & 0.458 \\
\hline 3 & HAP3 & $35 \mathrm{~nm}$ & 0.808 \\
\hline 4 & HAP4 & $57 \mathrm{~nm}$ & 0.861 \\
\hline
\end{tabular}

Kalaiselvi et al., 
The increment of calcinations temperature enhanced to degree of crystalline phase. Hydroxyapatite calcined at higher temperature shows no activity towards bioresorption and is insoluble in any physiological environment. This means that the high crystallinity of Hydroxyapatite gives teeth the chemical and corrosion resistance against the different $\mathrm{pH}$ conditions of saliva in mouth.

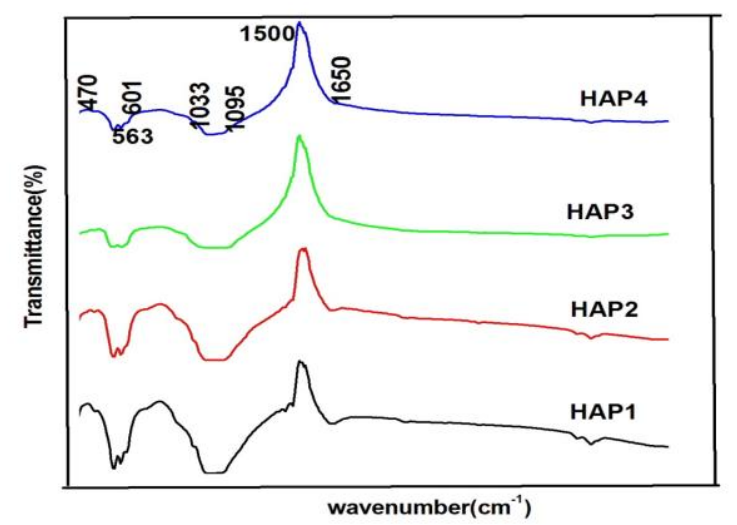

\section{Fig: 2 FT-IR spectra of HAP1, HAP2, HAP3 and HAP4} powders

Figure 2, shows the FT-IR spectra for HAP1, HAP2, HAP3 and HAP4 NPs in between the region of $4000-400 \mathrm{~cm}^{-1}$. The bands at $1650 \mathrm{~cm}^{-1}$ corresponding to $\mathrm{H}_{2} \mathrm{O}$ bending mode is more prominent in the case of HAP1, HAP2 as compared to Hydroxyapatite at HAP3 and HAP4.The two characteristic bands at $1033 \mathrm{~cm}^{-1}$ and $1095 \mathrm{~cm}^{-1}$ corresponding to stretching vibration of the phosphate group. The characteristic peaks at $601 \mathrm{~cm}^{-1}$ is due to $\mathrm{OH}^{-}$bending mode in the Hydroxyapatite lattice. The bands at 563 and 470 $\mathrm{cm}^{-1}$ corresponding to $\mathrm{PO}_{3}$ group which is present at calcined samples.

The $\mathrm{CO}_{3}{ }^{2-}$ related peaks such as $1420 \mathrm{~cm}^{-1}$ was disappeared due to the heat treatment. The peak at $3550 \mathrm{~cm}^{-1}$ is due to the presence of Hydroxyapatite. Generally stoichiometric Hydroxyapatite donot have water molecule in the lattice while non stoichiometric may have of water molecules in the lattice. It should be noted that absorption bands at 3581 $\mathrm{cm}^{-1}, 3561 \mathrm{~cm}^{-1}, 3487 \mathrm{~cm}^{-1}, 2430 \mathrm{~cm}^{-1}, 1283 \mathrm{~cm}^{-1}$ and 917 $\mathrm{cm}^{-1}$ distinguish the absence of octacalcium phosphate and confirm the Hydroxyapatite stoichiometry. The FT-IR spectra of HAP1, HAP2, HAP3 and HAP4 nanoparticles reveals that the bands corresponding to hydroxyl group decreases as the temperature increases where as the strength of $\mathrm{PO}_{4}{ }^{3-}$ stretching vibration peaks have increased with respect to increment of calcinations temperature.

The morphologies of HAP1, HAP2, HAP3 and HAP4 NPs were recorded by SEM are shown in figure 3(a-d). These samples mostly consist of the particles with agglomerated rod like structure, homogeneous and uniform distribution of components. The width and the length of the nano rods for different calcined samples are in the range of 47 to $155 \mathrm{~nm}$ and 240 to $878 \mathrm{~nm}$. This reveals that the length and the width of the nano rods changes as the temperature changes due to nucleation and growth of nanostructures which are attributed to relative surface energy of the nanoparticle. The surface energy determines the growth rate and morphology of the nanoparticle due to $\mathrm{OH}^{-}$ absorbance. The movement of $\mathrm{Ca}$ and $\mathrm{PO}_{4}$ in the samples will be restricted when $\mathrm{OH}^{-}$concentration increases. Due to the unrestricted movement of $\mathrm{Ca}$ and $\mathrm{PO}_{4}$ groups in the sample when the temperature increases the $\mathrm{OH}^{-}$ concentration decreases and length of the nanorod increases.

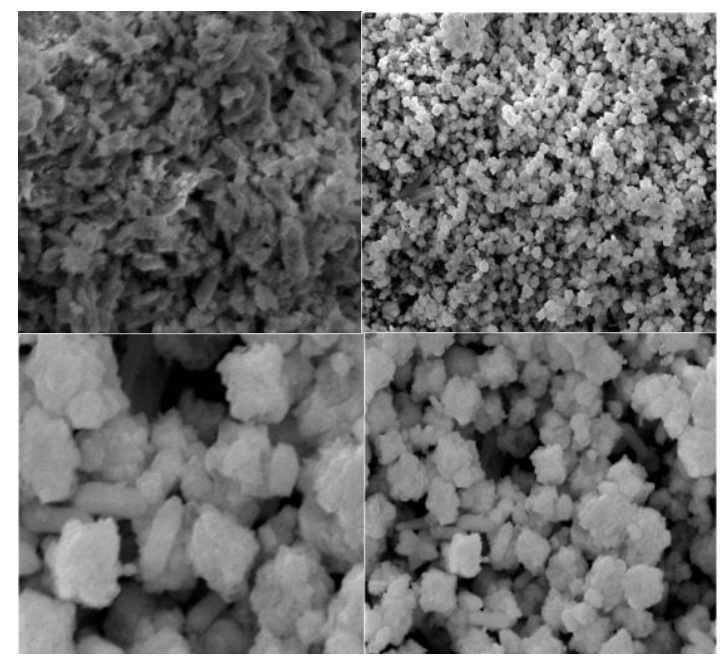

Fig: 3 SEM images of HAP1, HAP2, HAP3 and HAP4 powders.

\section{CONCLUSION}

The influence of calcinations temperature on the characteristics of Hydroxyapatite nanoparticles was studied from the samples synthesized by wet chemical method. The crystalline phase formations, presence of functional groups, morphological of prepared nanoparticles were investigated using XRD, FT-IR and SEM analysis. XRD investigation reveals that the crystallite size and crystallinity increased with increase the calcinations temperature. The FT-IR spectral analysis showed all the characteristic bands of Hydroxyapatite nanoparticles. A SEM study reveals the formation of agglomerated rod like structures which stimulate the morphology, size and phase of Hydroxyapatite crystals in human teeth.

\section{REFERENCES}

[1]. Haresh,M. Pandya., 2012. Modelling Scenario in Nanotechnology Today, Journal of Environmental Nano technology, 1(1), 01-04.

[2]. Amogh Tathe, Mangeshghodke, Anna pratimanikalje, 2010. A Brief Review: Biomaterials and their apllication, Int J pharmpharmsci, 2(4), 19-23.

[3]. HadedAlobeedallah, Jeffrey L.Ellis, 2011, Preparation of nanostructured Hydroxyapatite in organic solvents for clinical application, trends, Biomaterials. Artif. Organs, 25(1), 12-19.

Kalaiselvi et al., 
[4]. KapoorSeema, Batra Uma and KohliSuchita, 2011, Transformation in sol-gel synthesized nanoscale Hydroxyapatite calcined under different temperatures and Time conditions, Journal of Materials Engineering and performance, 21(8),1737-1743.

[5]. Sanosh, K. P., Min Cheolchu, Balakrishnan, A., Kim, T. N. and Seong Jai Cho., 2009, Preparation and characterization of Nano hydroxyapatite powder using sol gel technique, Bull Material. Sci., 32(5), 465470

[6]. Haresh, M., Pandya, P., Anitha, 2013,, Comprehensive Review of Preparation Methodologies of Nano Hydroxyapatite, J. Environ. Nanotech. 3(1)101-121.

[7]. Azade yelten, Suat yilma,2016,Various Parameters Affecting the Synthesis of the Hydroxyapatite Powders by the Wet Chemical Precipitation Technique, Materials Today, 3(9), 2869-2876,.

[8]. Gobi, D., Indira, J., Nithiya, S., Kavitha, L., Mchi Mudali, U. and Kanimozhi, K., 2013, Influence of surfactant concentration on nano hydroxyapatite growth, Bull. Mater. Sci., 36(5), 799-805.

[9]. Anitha,Haresh,P., Pandya,M., 2014, Synthesis, Characterization and Antimicrobial Activity of Nano Hydroxyapatite Via a Novel Sol Gel Method, Nanotechnology Research and Practice, 3(3)120-126.

[10]. Kalaiselvi,V and Mathammal,R, 2015, Effect of Titanium Dioxide Doping on Nano Hydroxyapatite synthesised from egg shells via wet chemical Method, International Journal \& Magazine of Engineering, Technology, Management and Research,3(8),133137.

[11]. Antonio Zanotto,Maria Luisa Saladino, Delia Chillura Martino, Eugenio caponetti, 2012, Influence of Temperature on Calcium Hydroxyapatite Nanopowders , Advances in Nanoparticles, 1, 21-28.

[12]. Naruporn Monmaturapoj, 2008, Nano-Size Hydroxyapatite Powders Preparation by WetChemical Precipitation Route, Journal of Metals, Materials and Minerals. 18(1), 15-20.

[13]. Mechay,A,, H. EL Feki, F. Schoenstein , Jouini,N., 2012, Nanocrystalline hydroxyapatite ceramics prepared by hydrolysis in polyol medium, Chemical Physics Letters, 541, 75-80.

[14]. Kristine Salma, Liga Berzina-Cimdina, Natalija Borodajenko, 2010, Calcium phosphate bioceramics prepared from wet chemically precipitated powders, Processing and Application of Ceramics 4,145-151. 